\title{
TEXTE LITTÉRAIRE DANS LA GLOTTODIDACTIQUE
}

\author{
KRYSTYNA ANTKOWIAK \\ University Lódź
}

ABSTRACT. Reading in a foreign language is a skill which is important for an overall competence. The researches done in the 1980s and the 1990 s proved the utility of the text and especially of the literary one in language teaching. The present article deals with the use of self instruction in language teaching. Self instruction aims not only at giving a learner some knowledge but also at making him able to learn by himself and at giving him an attitude of independence towards the society he lives in, which is possible only when he is aware of how a society works. Reading itself is an autonomous activity because it is a self-directed process of discovering the meaning of a text. In order to make this process efficient a learner-reader should learn some strategies which will enable him not to use a dictionary too often. Tasks in relation to reading texts should lead a learner to think over the meaning of the text, to compare his opinions with those of the author, to speak about his reactions. And last but not least: reading literary works makes a learner get acquainted with the culture of people whose language he is learning.

La compétence linguistique dans une langue étrangère suppose la capacité de lecture, d'écriture, d'expression orale et d'audition. Lors de la lecture, le contact avec une langue étrangère se concentre exclusivement sur le contenu; c'est pourquoi on la considère comme une importante capacité perceptive, essentielle pour la compétence linguistique globale ${ }^{l}$. Les profits de la lecture sont évidents: elle permet d'améliorer la connaissance du lexique car en lisant, l'apprenant non seulement se rappelle le vocabulaire déjà connu, mais aussi, il l'élargit en prenant conscience de nouveaux emplois des mots déjà rencontrés. La lecture permet de connaitre le fonctionnement du vocabulaire dans les contextes naturels. Les unités lexicales prennent alors pour l'apprenant le même sens qu'ils ont pour un native speaker. Il en est de même pour les structures grammaticales et syntaxiques. En ce qui concerne l'organisation d'un texte, le lecteur prend connaissance de la structure des unités plus développées que la phrase: paragra-

${ }^{1}$ Krashen S.D.; Terrel T.P. : The natural approach. Language acquisition in the classroom. Oxford, 1983; cité d'après: Halina Chodkiewicz, O sprawności czytania w nauczaniu języka obcego. Warszawa, 1986. 
phe, récit, description. Enfin, l'expression des sentiments et la description de faits illustrent le fonctionnement du lexique et de la grammaire dans la communication.

Il n'y a plus besoin de prouver l'utilité de la lecture dans la glottodidactique. Les apprenants eux-mêmes sont persuadés qu'il n'est pas possible d'apprendre une langue étrangère sans lire dans cette langue ${ }^{2}$. L'utilité de la lecture étant indiscutable il reste à savoir quel genre de texte est le plus utile. D'après les travaux publiés jusqu'à présent il paraît que c'est le texte littéraire qui constitue l'un des meilleurs matériaux didactiques ${ }^{3}$. A. Kozłowski ${ }^{4}$ affirme que la glottodidactique devrait s'intéresser particulièrement à la littérature parce que celle-ci contient des textes qui représentent les styles et les façons de communiquer de tout les groupes sociaux ${ }^{5}$, en plus, ils contiennent les lexiques universels et les constructions syntaxiques qui peuvent se réaliser dans toutes les situations de communication linguistique, ce qui fait que la littérature est "la source des modèles". Dans un texte littéraire l'énoncé apparaît dans un contexte aussi bien linguistique que situationnel, ce qui fait disparaître la différence entre la compréhension de l'oral et de l'écrit. D'où l'opinion qu'un texte glottodidactique devrait être construit comme un texte littéraire ${ }^{6}$.

Parmi les textes littéraires les plus utiles sont ceux de courte narration: un récit ou une nouvelle qui reflètent le mieux le vocabulaire actuel.

Cependant, un texte littéraire n'est pas qu'un phénomène linguistique, c'est aussi l'expression verbale de l'expérience humaine, ce qui provoque souvent une réaction affective spontanée et engage une conversation analogue aux situations authentiques de communication. Par la pluralité d'interprétations possibles, un texte littéraire plus que tout autre invite à s'exprimer ${ }^{7}$. Bien sûr, un texte littéraire ne peut être exploité que dans l'enseignement à des personnes dont le niveau de connaissance d'une langue est assez élevé. Les formes de travail sont nombreuses: on peut essayer de définir la spécificité et les caractéristiques des genres littéraires ou des moyens stylistiques; en ce cas-là on peut se référer à la connaissance de la littérature matemelle; on peut essayer de prouver ses opinions, de les comparer à celles des autres, de commenter, de présenter différents arguments. Comme un travail à domicile, on propose la lecture d'un texte et l'étude du vocabulaire. Au niveau avancé, une lecture silencieuse devrait être une forme principale de perception.

${ }^{2}$ Réné Labonté, Lire avec plaisir en français langue seconde. Dans: Le Français dans le Monde. No 144, IV 1979. 1979

${ }^{3}$ Voir surtout les articles dans Le Français dan sle Monde, $N^{\circ} 141$, XI-XII 1978 et $N^{\circ} 144$, IV

${ }^{4}$ Aleksander Kozłowski, Literatura piękna w nauczaniu jezzyków obcych. Lódź, 1991, p. 11.

${ }^{5}$ Ivo H., Allgemeine Lernziele des Literaturunterrichts, dans: Wilkending, G. (Réd.) Literaturunterricht, München, 1972, p. 174, cité d'après A. Kozłowski, op. cit.

' Leontjew A.A. Psycholinguistique und Sprachunterricht, Stuttgart, 1974, p. 52, cité d'après A. Kozłowski, op. cit.

${ }^{7}$ A. Kozlowski, op. cit., p. 36. 
L'utilité d'un texte littéraire étant déjà prouvée il serait intéressant de savoir comment on peut l'utiliser dans l'enseignement semi-autonome, c'est-àdire celui qui vise non seulement à faire acquérir des connaissances précises mais aussi à rendre un apprenant autonome - capable de s'instruire par luimême ${ }^{8}$ Cela signifie qu'il pourra définir ses besoins (ce qu'il ne sait pas, ce qu'il veut savoir, ce qui est utile), de même que les méthodes dont il veut se servir. L'enseignement en semi-autonomie vise à rendre l'apprenant indépendant de l'enseignant.

Cependant, ce n'est pas le seul but. L'enseignement en semi-autonomie est une approche d'un apprenant comme d'un individu humain conditionné par sa philosophie, son échelle de valeurs, ses idées et ses opinions, chez qui on veut former une attitude d'activité intellectuelle. Cette approche part du principe que l'apprentissage ne se fait pas seulement à l'école et son but est de former la personnalité - il s'agit de former un niveau intellectuel garantissant à l'individu l'indépendance par rapport à la société dans laquelle il vit, ce qui n'est possible que lorsque celui-ci comprend les mécanismes du monde qui l'entoure - ainsi l'enseignement en semi-autonomie devient-il préparation à la vie dans la société démocratique. L'insertion de la lecture littéraire dans l'enseignement en semiautonomie paraît d'autant plus justifiée qu'on a déjà signalé, en passant, ses propriétés autonomisantes. Qu'un élargissement de la compétence linguistique par la lecture se fait sans intervention des enseignants et que le profit dépend des connaissances préalables prouvent que la lecture peut participer à l'individualisation de l'enseignement. Il s'avère même que cette "acquisition sauvage" est plus efficace que l'étude systématique d'un manuel ${ }^{9}$. D'autres observations qui démontrent l'utilité d' un texte littéraire dans l'enseignement traditionnel contiennent des éléments importants pour celui en semi-autonomie: on s'est aperçu que la littérature agit sur l'affectivité, un lecteur engageant son intelligence et ses émotions lorsqu'un texte traite de ce qui est pour lui important ${ }^{10}$; par sa réaction au texte, l'apprenant connaît lui-même ce qui contribue au développement de sa personnalité. Puisque chaque lecture est une approche individuelle du sens à travers d'une interaction autogérée avec un discours ${ }^{11}$, elle constitue une forme fondamental de l'autonomie. Enfin, lecture individuelle étant un'élement important de ce qu'on appelle „culture de loisirs" réalise l'apprentissage hors de classe.

L'autonomie se réalisant par une activité consciente, la lecture dans le cadre de l'enseignement semi-autonome implique qu'un apprenant connaisse luimême en tant que lecteur. Il faut qu'il sache caractériser ses habitudes de lecture, sa motivation et ses intérêts aussi bien en langue étrangère qu'en mater-

\footnotetext{
${ }^{8}$ H. Holec, L'autonomie, Nancy, 1979.

${ }^{9}$ Réné Labonté, art. cité.

${ }^{10}$ Francine Cicurel Lecture de la nouvelle. Dans: Le Français dans le Monde, N ${ }^{\circ}$ 141, XI-XII 1978.

${ }^{11}$ Tricia Hedge Using Readers in Language Teaching, s. 1.: Macmillan Publishers Ltd 1985, p. 76.
} 
nelle; ensuite il faut qu'il soit aussi conscient de la notion de lecture: que veut dire „lire un texte", qu'est-ce qu'une vraie lecture ${ }^{12}$, etc. Pour l'aider à se définir comme lecteur on peut lui poser des questions portant sur ses habitudes de lecture en langue matemelle: quelle thématique l'intéresse, s'il lit des livres ou de la presse, s'il lit souvent, où et quand il lit. En vue d'évaluer ses capacités de lecture en langue étrangère, on peut lui poser des questions analogues, puis en ajouter d'autres sur le processus même de la lecture: s'il lit lentement, s'il traduit en lisant, quelles sont ses difficultés (par exemple: rythme trop lent de sa lecture, compréhension de mots nouveaux, compréhension du sens des phrases). Ensuite, pour l'aider à comprendre la nature même de la lecture, on lui demandera ce que veut dire, d'après lui, être un bon lecteur, si c'est: lire vite, comprendre tout les mots, deviner le sens, lire exactement tout,... Enfin, on peut lui demander s'il est possible d'apprendre à bien lire, à être un lecteur efficace, et comment. Quelles que soient les réponses données par l'apprenant, c'est à l'enseignant de lui faire comprendre à quelles exigences doit satisfaire la lecture pour être utile dans l'apprentissage. Avant tout, il faut qu'il comprenne que le but de la lecture n'est pas de reconnaître chaque unité lexicale mais de saisir le sens général de l'énoncé. La qualité de la lecture dépend de la vitesse et des stratégies appliquées.

On sait que la compréhension et la mémorisation d'un texte sont meilleures chez ceux qui lisent vite ${ }^{13}$. Le choix de stratégie implique une attitude active par rapport au texte ce qui veut dire qu'un lecteur doit déterminer sa façon de lire en fonction de la nature du texte, du but de la lecture, et en fonction de lus même, de ses besoins et du temps dont il dispose ${ }^{14}$. Puisqu'il est essentiel que la lecture ne soit pas trop lente, il faut munir un apprenant des capacités qui lui permettront de ne pas se servir trop souvent d'un dictionnaire; en voici les plus importantes:

- déduction du sens des mots inconnus d'après le contexte,

- compréhension des informations exprimées implicitement et explicitement,

- perception des relations entre les phrases et entre les fragments plus longs,

- distinction d'un fil conducteur des thèmes secondaires,

- perception de la fonction communicative des propositions et des expressions

Toutes ses capacités sont d'autant plus importantes qu'elles empêchent que la lecture devienne un déchiffrement pénible d'un texte, mot par mot. Or, pour contribuer au progrès dans l'apprentissage d'une langue étrangère la lecture doit

${ }^{12}$ La notion de la vraie lecture se trouve dans le livre de François Richaudeau, Sur la lecture, Paris, 1992, p. 153.

${ }^{13}$ François Richaudeau, op. cit., p. 154.

${ }^{14}$ François Richaudeau, op. cit., p. 175 
être authentique, c'est à dire motivée par le plaisir de lire ${ }^{15}$. Ainsi, les capacités mentionnées ci-dessus contribuent essentiellement à l'attitude individuelle d'un apprenant par rapport à la lecture. L'enseignement de lecture doit aboutir à la lecture extensive, c'est-à-dire individuelle, de longs textes, choisis librement, qu'on aborde de son propre gré, pour vivre une aventure ou acquérir une expérience; ce n'est qu'une telle lecture qui rend un apprenant autonome. Cette étape doit être précédée par la lecture intensive - des textes courts, guidée par l'enseignant et suivie des tâches dont l'acomplissement permettra d'acquérir l'indépendance envers le dictionnaire. Ce qui peut faciliter la déduction du sens d'après le contexte c'est l'exploitation des indices syntaxiques, logiques ou culturels pour deviner le sens des nouvelles unités lexicales, c'est aussi l'identification des rapports à l'intérieur de la phrase (sujet verbe) et la reconnaissance des relations entre les phrases. Ce qui peut aussi aider à saisir le sens général, c'est la capacité d'identifier le type d'organisation d'un texte du point de vue rhétorique (par exemple: l'idée principale complétée par des thèmes secondaires, l'ordre chronologique de présentation, la description, l'analogie, le contraste et la classification) ${ }^{16}$. Enfin, pour amener le lecteur à une compréhension plus exacte, il serait utile de lui poser des questions portant sur le contenu, mais il faut éviter celles auxquelles on peut donner des réponses simples et évidentes; les questions doivent amener à la réflexion et aux conclusions. Pour rendre le lecteur actif pendant la lecture on lui demandera de faire des exercices qui supposent l'emploi des informations trouvées dans le texte: les placer dans un diagramme, résoudre un problème posé avant la lecture, disposer les faits dans un ordre chronologique, remplir une grille, ce qui nécessite une autre disposition du contenu. D'autres tâches impliqueront l'emploi plus authentique des informations contenues dans le texte: rédaction d'une lettre, remplissement d'un formulaire, comparaison des plusieurs textes sur le même sujet. Le résumé ou la prise de notes font preuve de la compréhension du texte; c'est pourquoi l'élaboration de ces capacités doit être intégrée à l'enseignement de lecture ${ }^{17}$.

Mais avant même d'apprendre au lecteur toutes ces capacités, il faut former en lui l'attitude de confiance - on peut lui suggérer qu'il n'est pas toujours nécessaire de consulter un dictionnaire, qu'il est plus facile de saisir l'idée principale quand on n'interrompt pas la lecture, qu'il est possible de trouver le sens des mots nouveaux quand on lit un texte pour la deuxième fois. Enfin, il faut persuader l'apprenant qu'il accepte l'imprécision et l'insuffisance de la compréhension au début de la lecture. On peut aussi lui apprendre à vérifier la compréhension pendant la lecture par la réflexion sur ce qu'il a déjà compris,

\footnotetext{
${ }^{15}$ Raphaël Nataf, L'écrit littéraire. Dans: Le Français dans le Monde Nº 109, XII 1974.

${ }^{10}$ Jack C. Richards, New ways in Teaching Reading, TESOL, 1993.

${ }^{17}$ Françoise Grellet, Developping Reading Skills, C.U.P., 1994.
} 
l'anticipation de ce qui s'ensuit et la référence à ses connaissances et à ses expériences.

Puisque l'enseignement en semi-autonomie à pour but de développer la personnalité de celui qui apprend il ne suffit pas qu'il comprenne un texte; il faut aussi qu'il soit capable de l'évaluer, de dire ce qu'il en pense. Il faut donc qu'il sache distinguer dans un texte un fait d'une opinion, ce qui lui permettra d'apercevoir comment l'auteur essaie d'influencer les idées du lecteur. Il faut le rendre sensible au ton du texte: objectif ou subjectif (ironie, réprobation, approbation, persuasion).

L'autonomie du lecteur suppose son indépendance par rapport au texte, surtout lorsqu'il s'agit d'un texte littéraire. Il faut qu'un apprenant sache comment ne pas se laisser entraîner par la fiction littéraire, comment découvrir le procédé artistique par lequel l'écrivain a atteint l'effet souhaité. Lors de la discussion on attirera l'attention d'un apprenant aux valeurs éthiques du texte, à la richesse du contenu, on lui en fera découvrir toute la portée.

Même si la lecture extensive est une lecture individuelle, cela ne veut pas dire qu'un lecteur-apprenant est laissé à lui-même. L'intérêt pour la lecture croît lorsqu'on partage avec les autres ses opinions et qu'on parle de ses expériences. C'est pourquoi le texte lu hors de classe devrait être l'objet du cours pendant lequel les apprenants pourront exprimer oralement ou par écrit, leurs réactions aussi bien intellectuelles qu'émotionnelles. Il est important qu'ils ne se limitent pas au résumé ou au simple récit. La présentation des opinions ne doit pas se faire au hasard. Chaque énoncé, même le plus court, doit être organisé d'après un schéma établi. L'opinion la plus simple au sujet d'un texte est l'approbation ou la réprobation qu'on peut présenter sous forme d'un rapport contenant des parties suivantes:

- introduction: ,récemment, j'ai lu un livre (titre, auteur, genre) que j'ai / je n'ai pas aimé,

- développement: un court résumé (protagonistes, intrigue); on peut s'abstenir de révéler la fin,

- conclusion: les arguments en faveur ou contre le livre.

Une autre forme du travail sur un texte est une interview suivie de la rédaction d'un compte-rendu; les questions doivent avant tout amener le lecteur à dire ses réactions. Pour que la lecture soit l'occasion de perfectionner l'expression écrite, on peut imposer la rédaction d'un rapport écrit. Celui-ci, à part des éléments ci-dessus, devrait contenir aussi des observations concernant le processus même de la lecture: le temps nécessaire, les difficultés, les expressions et les mots nouveaux rencontrés dans le texte, la contribution du texte à l'amélioration de la compétence linguistique. De telles observations permettront au lecteur d'évaluer sa compétence de lecture et le prépareront à l'apprentissage auto- 
nome. Il faut que les apprenants pensent, parlent et écrivent de ce qu'ils lisent, avant, pendant et après la lecture. L'expression écrite est particulièrement importante parce qu'elle nécessite une réflexion plus approfondie aussi bien sur ce qu'on lit et ce qu'on veut en écrire que sur l'aspect linguistique de l'énoncé. C'est pourquoi on peut conseiller aux apprenants de rédiger un journal de lecture; il ne s'agit pas de prendre des notes ou de faire des résumés. L'essentiel est de dépasser la problématique du texte. Cependant, ce journal ne peut pas être une improvisation. L'enseignant doit préparer des règles générales: longueur des paragraphes, critères d'évaluation; il peut aussi suggérer le contenu du journal: description d'un événement ou présentation d'un thème ou d'une idée particulièrement intéressants, justifier son choix; référer ce qu'on a lu à ses expériences ou à ses opinions; décrire un événement vécu analogue à celui dont parle le texte; expression des opinions, des observations, des réflexions provoquées par la lecture; évaluation du processus de lecture et de son utilité pour la compétence linguistique ${ }^{18}$.

On a déjà dit plusieurs fois que l'enseignement en semi-autonomie vise la formation de la personnalité et de l'intelligence d'un apprenant; qu'il veut éveiller en lui la curiosité intellectuelle, élargir ses intérêts et lui apprendre à approfondir ses connaissances. C'est pourquoi les textes étudiés en classe ne doivent pas traiter seulement de ce qui intéresse un apprenant. 1 l faut qu'ils lui ouvrent de nouvelles perspectives, surtout celles sur le pays dont il apprend la langue: la culture, la géographie, l'histoire et l'actualité. Toute langue est l'expression de la culture d' une nation, c'est pourquoi il faut que l'apprentissage d'une langue étrangère soit l'occasion de faire connaissance avec ce qui jusqu'alors était inconnu, de s'ouvrir à ceux qui jusqu'alors étaient les autres et de rendre familier ce qui était étranger. Dans cette perspective l'enseignement d'une langue étrangère peut devenir préparation à la vie dans l'Europe unie. En vue de cette préparation il y a une autre catégorie de textes qu'on devrait aborder lors des classes de langue: ce sont les textes qui traitent des problèmes internationaux importants et d'actualité: défense de droits de l'homme, écologie, etc.

Un cours de lecture devrait être un progrès en compétence linguistique, en connaissance du monde, des autres et de soi-même.

\section{BIBLIOGRAPHIE}

Dickinson L., Self instruction in Language Teaching, C.U.P., 1987.

Holbrook D., English for Maturity, C.U.P.,1961.

Littérature et classe de langue. Réd. H. Besse, Paris, 1982.

${ }^{18}$ Jack C. Richards, op. cit. 